\title{
OUTCOMES OF NEONATAL CHYLOUS EFFUSIONS: A 20-YEAR WEST-AUSTRALIAN TERTIARY CENTER EXPERIENCE
}

\author{
A. Anvekar, G. Athalye-Jape, H. Panchal, S. Rao, R. Kohan
}

Department of Neonatal Paediatrics (AA), Fiona Stanley Hospital, Perth; Department of Neonatal Paediatrics (HP,SR), Perth Children Hospital, Perth; Department of Neonatal Paediatrics (GJ,RK), King Edward Memorial Hospital, Perth, Australia

\section{ABSTRACT}

Neonatal chylous effusions are rare entity with limited evidence-based management. We conducted a retrospective review of neonates admitted to King Edward Memorial and Princess Margaret/Perth Children's Hospital over 20 years with laboratory-confirmed chylous effusions. A total of 51 infants with chylous effusion were identified. Median gestational age and birth weight were 35.5 weeks and 2620 grams respectively. Congenital [27/ 51] and acquired [24/51] cases were included. Antenatal interventions were performed in 17/22 with antenatal hydrops and 50/51 needed postnatal drains. Effusions were monitored with serial ( $\geq 2$ ) chest ultrasounds in 29/51 infants and multiple ( $\geq 5$ ) $x$-rays in 45/51 infants. Median duration of mechanical ventilation, oxygen requirement, and hospital stay was 294.5 hours, 400 hours, and 49 days respectively. 39/51 received medium chain triglyceride (MCT) diet while 8/51 received octreotide. Six infants died during hospital stay. 12/19 had normal developmental assessment at one-year. The acquired group had higher number of $x$ rays done, need for MCT diet and inotropes, and hospital stay vs congenital group. Duration of drains, radiological investigations and immunoglobulin administration were higher in neonates who received octreotide. Syndromic association, duration of ventilation and oxygenation were risk factors for mortality. In our setting, neonatal chylous effusions are asso- ciated with significant morbidity and mortality.

Keywords: neonate, chylous effusion, octreotide

Chylous effusions in the neonatal period are rare. Chylothorax is the collection of chyle in the pleural space (1). Neonatal chylothorax can be congenital or acquired (2) with abnormalities in the lymphatic system occurring spontaneously or in conjunction with congenital anomalies contributing to congenital chylothorax (3). Congenital chylothorax is the most common etiology of neonatal pleural effusions (4). The incidence of congenital chylothorax has been estimated at 1 in 24,000 live births in a population-based study (5) and is responsible for 1 in 2,000 neonatal intensive care unit (NICU) admissions (6). Acquired chylothorax is usually due to trauma to the lymphatic vessels or thoracic duct and occasionally due to venous hypertension or venous thrombosis (7). Most cases of acquired chylothorax are postoperative (8). Chylous ascites is the accumulation of chyle in the peritoneal cavity with an incidence of 1 in 20,000 admissions for primary chylous ascites (9). Chylo-pericardium is extremely rare (10-12). Chylous effusions lead to significant mortality and morbidity such as nutritional deficiencies, respiratory compromise, lymphopenia, immunosuppression, and prolonged hospitalization (13-15).

Antenatal interventions such as amnio- 
reduction, fetal thoracentesis and thoracoamniotic shunts have been used to treat fetal effusions (16). Postnatal interventions including use of total parental nutrition (TPN), medium chain triglyceride (MCT) formula, octreotide, somatostatin, and surgical management have been reported as management options to treat chylous effusion (16).

We aimed to describe our experience in management of neonates with chylous effusions admitted in our NICU at King Edward Memorial hospital (KEMH) and Princess Margaret hospital (PMH)/ Perth Children's Hospital (PCH) over 20 years (2000-2020), including developmental follow-up at one year of age. Furthermore, we compared congenital vs acquired chylous effusions, the use of octreotide, and evaluated risk factors for mortality.

\section{MATERIAL AND METHODS}

This retrospective study was conducted at the tertiary NICUs at KEMH and PMH/ $\mathrm{PCH}$ in Western Australia. The study is approved by the institutional ethics committee as a quality assurance project (5055).

\section{Participants:}

\section{Inclusion criteria:}

All neonates admitted to KEMH and PMH/PCH NICU between January 2000 and December 2020 with a confirmed laboratory diagnosis $(2,17,18)$ of chylous effusion were eligible for inclusion in the study. Diagnosis of chylous effusion was confirmed with laboratory analysis of the effusion showing: (1) a triglyceride level $>1.1 \mathrm{mmol} / \mathrm{L}$ (with enteral fat intake) or, (2) a cell count $>1000 / \mathrm{mL}$ with a lymphocyte fraction of $>80 \%(2,17,18)$. Exclusion criteria: Infants who did not fulfil the laboratory criteria for chylous effusion.

\section{Outcomes of interest:}

(1) Investigations and Interventions: Prenatal investigations and interventions, details of postnatal drains and imaging studies, medical and surgical interventions for chylous effu- sion and genetic testing.

(2) Clinical: duration of ventilator support and oxygen supplementation, type of milk used for enteral feeding, length of NICU stay, incidence of proven late onset sepsis (LOS), mortality and neuro-developmental outcomes at one year of age using a Griffiths Mental Developmental Scale assessment [Griffiths-II, or GMDS-ER (extended revised), or Griffiths-III depending on epoch].

\section{Pre-planned subgroups:}

(1) Congenital vs acquired; (2) Those who received octreotide vs. those who did not; and (3) Risk factors for mortality.

Methods: Our hospital electronic neonatal database (NeoBase) was searched to find full-term and preterm infants with a diagnosis of chylous effusion.

Data collection: Data was collected from the patient medical records and NeoBase using a standardized, pre-piloted form and entered into a password protected excel worksheet after de-identification. Data collected included key neonatal demographics, and other pre-defined focused outcome data.

Statistical methods: Statistical analysis was done using Stata 16.0 (StataCorp. 2019. Stata Statistical Software: Release 16. College Station, TX: StataCorp LLC). Since the continuous variables were not normally distributed, they were summarized using median and interquartile range (IQR). Categorical variables were expressed as frequency and percentage. Wilcoxon rank sum test was used to compare non-parametric data. Fisher's exact test was used for analysis of contingency tables. Univariable logistic regression model was carried out to evaluate the association between various risk factors and neonatal mortality; unadjusted odds ratios and $95 \%$ confidence interval (CI) were reported for this analysis. For all analyses, a two-tailed $\mathrm{p}<0.05$ was considered statistically significant.

RESULTS 


\section{TABLE 1}

Demographics and Outcomes of All Study Neonates with Chylous Effusion

\begin{tabular}{|c|c|}
\hline Variable & $\mathbf{n}=\mathbf{5 1}$ \\
\hline Gestational age (weeks) & $35.5(34-37.2)$ \\
\hline Birth weight (grams) & $2620(2270-3092)$ \\
\hline $\begin{array}{l}\text { Gender } \\
\text { Male } \\
\text { Female }\end{array}$ & $\begin{array}{l}32(62.74 \%) \\
19(37.26 \%)\end{array}$ \\
\hline Antenatal hydrops & $22(43.14 \%)$ \\
\hline Antenatal intervention & $17(32.69 \%)$ \\
\hline $\begin{array}{l}\text { Mode of delivery } \\
\text { Vaginal } \\
\text { Assisted vaginal } \\
\text { Caesarean section }\end{array}$ & $\begin{array}{l}16(31.37 \%) \\
5(9.8 \%) \\
30(58.82 \%)\end{array}$ \\
\hline $\begin{array}{l}\text { Duration of mechanical } \\
\text { ventilation (hours) }\end{array}$ & $\begin{array}{l}294.5(96.25- \\
633.75)\end{array}$ \\
\hline Duration of CPAP (hours) & $104(16-399)$ \\
\hline Duration of oxygen (hours) & $400(94-1026)$ \\
\hline Inhaled nitric oxide & $13(25.49 \%)$ \\
\hline Number of drains & $4(2-9.75)$ \\
\hline Duration of drains (days) & $14.5(10-28.25)$ \\
\hline Number of $x$-rays & $18(7.25-25.5)$ \\
\hline $\begin{array}{l}\text { Number of chest } \\
\text { ultrasound }\end{array}$ & $2(0-4)$ \\
\hline Nil by mouth (days) $(n=33)$ & $12(5-18.25)$ \\
\hline Received MCT formula & $39(76.47 \%)$ \\
\hline $\begin{array}{l}\text { Discharged on MCT } \\
\text { formula }\end{array}$ & $13(25.49 \%)$ \\
\hline Octreotide used & $8(15.69 \%)$ \\
\hline Surgical intervention & $0(0 \%)$ \\
\hline Stay in NICU (days) & $49(27.75-68)$ \\
\hline Mortality & $6(11.76 \%)$ \\
\hline
\end{tabular}

A total of 54 neonates were identified from NeoBase with a broad diagnosis of effusion. Three were excluded due to not satisfying criteria for laboratory diagnosis of chylous effusion resulting in 51 infants for analysis.

Median (IQR) gestational age and birth weight were 35.5 (34-37.4) weeks and 2620 (2270-3092) grams respectively. Majority of the infants $45 / 51(88.2 \%$ ) were inborn. Majority were male [32/51(62.7\%) vs female: $19 / 51$ $(37.3 \%)]$. Twenty-one infants were delivered vaginally, of which five were assisted (ventouse: 3 , forceps: 2 ); thirty were delivered by caesarean section. Intubation at birth was needed for 27 infants, out of which seven required extensive resuscitation (external cardiac massage and/or adrenaline). Table 1 summarizes the neonatal demographics and outcomes.

\section{Investigations and Interventions}

Isolated chylothorax was present in $\mathbf{4 6}$ infants, chylothorax with chylous ascites was present in three neonates, chylothorax with chylous pericardium in one neonate, and one had isolated chylous ascites. Antenatal diagnosis of hydrops was established in 22 neonates. Fetal intervention procedures were performed in 17/22 neonates with hydrops. These were: amnio-reduction (7/17), thoracentesis (1/17), thoraco-amniotic shunt placement (3/ 17), amnio-reduction and thoracentesis (3/17), amnio-reduction with thoraco-amniotic shunt placement (2/17), and a combination of amnio-reduction, thoracentesis, and thoraco-amniotic shunt (1/17). Congenital chylous effusion was present in 27/51 (53\%) and 24/51 $(47 \%)$ infants had acquired chylous effusion. Acquired chylous effusion $(n=24)$ was seen following cardiac surgery $(n=12)$, tracheo-esophageal fistula repair $(n=5)$, congenital diaphragmatic hernia repair $(n=6)$ and post extracorporeal membrane oxygenation $(n=1)$.

Postnatal drains for pleural effusion were inserted in 50/51 (98.03\%) infants; and a median of four chest drains were inserted during their NICU stay. The median (IQR) number of days for chest drains and therefore the need for analgesia was 14.5(10-28.25) days.

In terms of diagnostic imaging, 88\% (45/ 51) of infants required multiple $x$-rays (average $\geq 5$ ) [median (IQR) 18 (7.25-25.5)] for monitoring pleural effusion. Serial ultrasound scans (average $\geq 2$ ) were performed in $57 \%$ infants (29/51) for assessment of effusion. None required surgical intervention for persistent chylous effusion.

Fourteen infants had low gamma globulin levels (serum IgG $<2 \mathrm{~g} / \mathrm{L}$ ) and received immunoglobulin infusion. 
Fourteen infants $(27.5 \%)$ had proven syndromic association [VATER/ VACTERL spectrum, CHARGE association, Down's syndrome, Turner's syndrome, Noonan syndrome, $8 \mathrm{p} \mathrm{mi-}$ crodeletion, pulmonary lymphangiectasis with loss in chromosome 7p22.2, PHACE, lymphedema distichiasis syndrome, myotubular myopathy] and 5/51 (9.8\%) couldn't be classified but had multiple congenital anomalies.

\section{Clinical Findings}

Median (IQR) duration of mechanical ventilation was 294.5 (96.25-633.75) hours and that of continuous positive airway pressure (CPAP) was 104 (16-399) hours. Median (IQR) duration of oxygen therapy was 400 (94-1026) hours. Thirty-two infants needed inotrope support for hemodynamic stability. Median (IQR) NICU stay was 49 (27.75-68) days. Overall survival was $90.2 \%$ (46/51).

Median duration where neonates received no enteral nutrition $(\mathrm{n}=33)$ was $12(5$ 18.25) days. Overall, $39 / 51$ infants $(76.47 \%)$ received MCT diet and 13/39 (33.33\%) of them were discharged home on MCT diet. Eight (24.24\%) infants required treatment with octreotide for persistent chyle in pleural space. None received somatostatin. Culture positive LOS was seen in 13/51 (25.49\%) infants.

None of the infants in our cohort had recurrence of chylous effusion. 29/46 (63\%) survivors were eligible for developmental assessment at $\mathbf{1 2}$ months corrected age. Of the 29, four infants died and six were lost to follow up. Hence, 19 infants received developmental assessments at one year. Of them, 12 had a normal developmental assessment; whereas remainder showed global developmental delay $(3 / 19 ; 16 \%)$, isolated gross motor delay $(1 / 19$; $5.3 \%)$, dystonic spastic cerebral palsy (1/19; $5.3 \%)$, autism spectrum disorder (1/19; 5.3\%), and deafness needing bilateral hearing aids $(1 / 19 ; 5.3 \%)$.

\section{Pre-planned subgroup analysis}

(1) Congenital vs. Acquired chylothorax: Number of $\mathrm{x}$-rays $(\mathrm{p}=0.047)$, infants who received MCT formula $(p=0.02)$, inotropic support ( $\mathrm{p}=0.001)$, and duration of stay in the NICU ( $p=0.026)$ were significantly higher in the acquired group (Table 2).

(2) Octreotide received vs. not: Duration of drains $(p=0.002)$, number of $x$-rays $(p=0.01)$, number of chest ultrasounds $(\mathrm{p}=0.001)$, and need for immunoglobulin $(\mathrm{p}=\mathbf{0 . 0 3})$ were higher in the octreotide group (Table 3).

(3) Survivors vs. non-survivors: Proven syndromic association $(\mathrm{p}=\mathbf{0 . 0 1})$, duration of ventilation $(\mathrm{p}=0.02)$, and oxygenation $(\mathrm{p}=0.03)$ were significant risk factors for mortality $(\mathrm{p}=0.01)$. No other variables were significant (Table 4).

\section{DISCUSSION}

Of the 51 infants admitted with chylous effusions to our tertiary referral NICU over a twenty-year period (2000-2020), half of them (53\%) had congenital chylous effusions. The acquired group showed increased need for radio-imaging, feeding with MCT formula, inotropic support and longer hospital stay. The sub-group of infants who received octreotide for persistent chylothorax management needed increased duration of drains, radioimaging frequency, and immunoglobulin infusions. Mortality was higher in those infants who had a pre-existing syndrome and in those needing prolonged respiratory and oxygen support.

Antenatal hydrops was diagnosed in 22 infants, and 17 of them underwent antenatal interventions. Antenatal hydrops and antenatal interventions had no significant association with mortality on logistic regression in our study. Al Tawai et al (19) reported $100 \%$ survival in all infants $(n=19)$ diagnosed with antenatal hydrops and who underwent antenatal procedures; however in another case series of 32 infants, Caserio et al (20) reported poor outcome with antenatal hydrops and $67 \%$ survival in those who needed antenatal shunts. Dorsi et al (21) reviewed prenatal factors associated with neonatal survival in $\mathbf{5 0}$ infants with congenital chylothorax. Hydrops fetalis and amnioreduction were not associated with mortality and reversal of hydrops following insertion of thoracoamniotic shunt was 


\begin{tabular}{|c|c|c|c|}
\hline \multicolumn{4}{|c|}{$\begin{array}{c}\text { TABLE } 2 \\
\text { Comparison of Patients with Congenital or Acquired Chylous Effusion }\end{array}$} \\
\hline Variable & Congenital $(n=27)$ & Acquired (n=24) & p-value \\
\hline Gestational age (weeks) & $35.2(34-36.5)$ & $36.1(34.2-37.5)$ & 0.2460 \\
\hline Birth weight (grams) & $2840(2300-3364)$ & $2550(2110-2895)$ & 0.1081 \\
\hline Antenatal hydrops & $22(81.48 \%)$ & $0(0 \%)$ & 0.00 \\
\hline Antenatal procedures & $15(55.56 \%)$ & $2(8.33 \%)$ & 0.00 \\
\hline Duration of mechanical ventilation (hours) & $223(45-534)$ & $293(98-637)$ & 0.24 \\
\hline Duration of CPAP (hours) & $105(21-264)$ & $168(6-474)$ & 0.55 \\
\hline Duration of oxygen (hours) & $314(9-691)$ & $484(208-1130)$ & 0.058 \\
\hline Inhaled nitric oxide (days) & $7(25.93 \%)$ & $6(25 \%)$ & 1.00 \\
\hline Number of drains & $13(10-26)$ & $20(10-32)$ & 0.82 \\
\hline Duration of drains (days) & $4(2-9)$ & $4(2-15)$ & 0.52 \\
\hline Number of $x$-rays & $13(6-23)$ & $22(12-30)$ & 0.047 \\
\hline Number of chest ultrasound & $1(0-3)$ & $3(0.5-4)$ & 0.25 \\
\hline Received MCT formula & $17(62.96 \%)$ & $22(91.67 \%)$ & 0.02 \\
\hline Discharged on MCT formula & $4(14.81 \%)$ & $9(37.50 \%)$ & 0.10 \\
\hline Octreotide & $6(22.22 \%)$ & $2(8.33 \%)$ & 0.25 \\
\hline Immunoglobulin & $9(33.33 \%)$ & $5(20.83 \%)$ & 0.36 \\
\hline Late onset sepsis & $6(22.22 \%)$ & $7(29.17 \%)$ & 0.74 \\
\hline Inotropes & $11(40.74 \%)$ & $21(87.50 \%)$ & 0.001 \\
\hline Proven syndromic association & $6(22.2 \%)$ & $8(33.3 \%)$ & 0.531 \\
\hline Total NICU stay (days) & $45(18-61)$ & $60.5(47-95)$ & 0.026 \\
\hline Mortality & $3(11.11 \%)$ & $3(12.50 \%)$ & 1.00 \\
\hline
\end{tabular}

\begin{tabular}{|c|c|c|c|}
\hline \multicolumn{4}{|c|}{$\begin{array}{c}\text { TABLE } 3 \\
\text { Comparison of Infants with Chylous Efiusion Treated or Not Treated with Octreotide }\end{array}$} \\
\hline Variable & Octreotide $(n=8)$ & No Octreotide $(n=43)$ & p-value \\
\hline Gestational age (weeks) & $34.3(33.05-38.05)$ & $35.6(34-37.3)$ & 0.58 \\
\hline Birth weight (grams) & $2900(2045-3462.5)$ & $2580(2240-3085)$ & 0.64 \\
\hline Antenatal hydrops & $5(62.5 \%)$ & $17(39.53 \%)$ & 0.268 \\
\hline Antenatal procedures & $4(50 \%)$ & $13(30.23 \%)$ & 0.416 \\
\hline Duration of mechanical ventilation (hours) & $631(287-724.5)$ & $194(64-484)$ & 0.08 \\
\hline Duration of CPAP (hours) & $431(153.5-1356)$ & $104.5(16-250)$ & 0.07 \\
\hline Duration of oxygen (hours) & $566(305-1395)$ & $389(75-768)$ & 0.20 \\
\hline Inhaled nitric oxide (days) & $1(12.5 \%)$ & $12(27.91 \%)$ & 0.66 \\
\hline Number of drains & $5(3.5-6.5)$ & $4(2-11)$ & 0.77 \\
\hline Duration of drains (days) & $38.5(23-50)$ & $13(8-23)$ & 0.002 \\
\hline Number of x-rays & $27.5(18-39.5)$ & $15(7-23)$ & 0.01 \\
\hline Number of chest ultrasound & $6(3.5-9.5)$ & $2(0-3)$ & 0.001 \\
\hline $\begin{array}{l}\text { Congenital } \\
\text { Acquired }\end{array}$ & $\begin{array}{l}6(75 \%) \\
2(25 \%)\end{array}$ & $\begin{array}{l}21(48.84 \%) \\
22(51.16 \%)\end{array}$ & 0.255 \\
\hline Received MCT formula & $8(100 \%)$ & $31(72.09 \%)$ & 0.17 \\
\hline Discharged on MCT formula & $3(37.50 \%)$ & $10(23.26 \%)$ & 0.40 \\
\hline Late onset sepsis & $3(37.5 \%)$ & $10(23.26 \%)$ & 0.4 \\
\hline Inotropes & $3(37.5 \%)$ & $29(67.4 \%)$ & 0.13 \\
\hline Immunoglobulin & $6(75 \%)$ & $8(18.60 \%)$ & 0.03 \\
\hline Proven syndromic association & $2(20 \%)$ & $12(27.91 \%)$ & 1.00 \\
\hline Total NICU stay (days) & $68.5(54.5-118.5)$ & $49(25-65)$ & 0.07 \\
\hline Mortality & $1(12.5 \%)$ & $5(11.63 \%)$ & 1.00 \\
\hline
\end{tabular}




\begin{tabular}{|c|c|c|}
\hline \multicolumn{3}{|c|}{$\begin{array}{l}\text { TABLE } 4 \\
\text { Risk Factors for Neonatal Mortality in Neonates with Chylous Effusion in the Study }\end{array}$} \\
\hline Variable & Odds ratio (95\% CI) & p-value \\
\hline Gestational age & $1.13(0.78-1.64)$ & 0.51 \\
\hline Birth weight & $0.99(0.99-1.0)$ & 0.33 \\
\hline Antenatal hydrops & $4.37(0.47-40.50)$ & 0.19 \\
\hline $\begin{array}{l}\text { Antenatal procedures (combined) } \\
\text { Amnioreduction } \\
\text { Thoracocentesis or Thoracoamniotic shunt }\end{array}$ & $\begin{array}{l}0.36(0.39-3.38) \\
0.97(0.09-9.83) \\
\text { Not calculable since zero event in one group }\end{array}$ & $\begin{array}{l}0.37 \\
0.97 \\
1.00 \\
\end{array}$ \\
\hline Congenital/Acquired effusion & $1.14(0.21-6.28)$ & 0.88 \\
\hline Duration of ventilation & $1.00(1.0004-1.0057)$ & $\mathbf{0 . 0 2}$ \\
\hline Duration of oxygen & $1.00(1.00009-1.00235)$ & $\mathbf{0 . 0 3}$ \\
\hline Octreotide used & $1.08(0.11-10.76)$ & 0.94 \\
\hline Proven syndromic association & $20(2.07-193.18)$ & 0.01 \\
\hline
\end{tabular}

associated with decreased mortality $(\mathrm{p}=0.001)$ (21). However, it is important to note that fetal interventions for hydrops are not risk-free (2224). Management of small to moderate pleural effusions using a conservative approach and considering fetal interventions for large effusions or extensive hydrops would potentially constitute a pragmatic clinical approach in the absence of large randomized studies.

Our cohort needed significant respiratory support in terms of ventilation and insertion of postnatal chest drains. Similar reports were published by other authors $(6,19,20,25-27)$. Exposure to radiographic imaging was higher in our study, which has its own side effects and costs related to comprehensive health care, although advances in imaging techniques has reduced the risk. None of the infants in our cohort had coagulopathy. Hypercoagulability and thrombosis attributed to antithrombin loss in chyle, have been reported in previous publications (28).

Medical management of chylous effusions is focused on decreasing the volume by with-holding enteral nutrition and providing TPN, using MCT formulas and somatostatin and its analogue octreotide. TPN and MCT formulas are recommended with worsening of effusion after starting enteral feeds (20). Ventilated or significantly unwell neonates are managed with TPN (29). Enteral feeding with MCT formulas may be considered in eligible infants. These bypass intestinal lymphatics and get absorbed directly into portal vein system (30). Median duration of withholding enteral feeds in our study $(n=33)$ was 12 (518.25) days. Thirty-nine infants $(76.47 \%)$ received MCT diet and 13/39 (33.33\%) of them were discharged home on MCT formula. There was no recurrence of chylous effusion reported in our cohort at follow up. Resch et al reported no recurrence of chylothorax at a mean age of seven years in a small cohort of six infants with congenital chylothorax (31).

Our cohort contains cases of both, congenital and acquired chylous effusion and we compared the two groups. Acquired chylous effusion was mostly postoperative except in one infant. Chylous effusion further adds to complications associated with surgery (27). Takahashi et al reviewed the outcomes of congenital and acquired chylothorax in their unit over a period of 22 years. A total of 28 infants were included of which 18 were congenital and 10 acquired. Only age at onset and laterality of chylothorax was different between the two groups. There was no difference noted in chylothorax resolution secondary to use of conservative treatment (MCT formula, octreotide, and steroids) vs. surgical therapy (32). 
We noted a higher use of MCT formula and NICU stay in the acquired group. There was no difference in octreotide use between the congenital and acquired groups and none needed surgery. The acquired group needed more immunoglobulin infusion and had higher number of $x$-rays.

Octreotide is a somatostatin analogue that reduces lymphatic flow by multiple mechanisms (33). It has been used as a second line agent in chylous effusion $(24,34)$. A systematic review reported its efficacy in $47 \%$ cases of chylothorax (8). Eight infants in our cohort were treated with octreotide. Duration of drains and frequency of radiological investigations were significantly higher in neonates who needed octreotide, suggesting higher severity of illness. Bialkowski et al (6) had also noted longer duration of pleural drainage in neonates who received octreotide. Although octreotide was effective in reducing chylous effusions (8), large prospective studies with focus on timing and duration of its administration (adequate case selection) are needed to confirm the findings.

The overall mortality rate in our study was $11.76 \%$, and using logistic regression, this was significantly higher in those with an underlying syndrome. In their case series of 33 infants with congenital chylothorax, Belini et al reported $67 \%$ survival, with six deaths in patients with lymphatic dysplasia and five deaths in those with a chromosomal abnormality (1). Caseria et al attributed the higher mortality in their series to inclusion of hydropic patients with associated major defects compared to other series (20). The presence of associated abnormalities is thus an important prognostic factor to be included in antenatal and postnatal discussions with parents/carers. In our cohort, duration of invasive ventilation and need for oxygen was an additional risk factor for mortality suggesting increased severity of illness.

Finally, it is important to discuss lymphatic imaging and recent advances in management of chylous effusion. Lymphatic imaging has been studied extensively in adults (3539). Lymphangiography has been the mainstay of lymphatic imaging which involves in- jection of dye into the peripheral lymphatics or lymph node followed by fluoroscopy/computer tomography (CT) to visualize the lymphatic system. This test has an advantage of being both diagnostic and therapeutic. Lymphatic scintigraphy has shown to be a reasonable alternative to other forms of lymphatic imaging and involves subcutaneous injection of radiotracer Technetium-99 albumin solution followed by imaging with CT/ single photon emission computer tomography (SPECT). Other modalities for lymphatic imaging are magnetic resonance (MR) lymphangiography and CT scan. Lymphatic imaging has been studied in the newborn and paediatric population (40-42). Bellini et al concluded that lymphoscintigraphy was safe and useful for diagnosing congenital lymphatic dysplasia in $\mathbf{3 1}$ neonates and children (40). Mihara et al performed indocyanine green lymphography in ten neonates with lymphatic dysplasia and demonstrated grades of severity (41). Dynamic contrast MR lymphangiography was performed in 35 neonates with pulmonary lymphatic abnormalities. These were classified as either neonatal chylothorax if they had pulmonary lymphatic perfusion syndrome or central lymphatic flow disorder (CLFD) if they had multiple flow abnormalities. The CLFD group showed higher mortality and morbidity (42). Lymphatic imaging could hence play an important role in diagnosis of lymphatic dysplasia in neonates and in management of refractory chylous effusions.

Several treatment options are emerging for the management of chylous effusion. Sirolimus (43) through its lymphangiogenesis inhibitory action by decreasing vascular endothelial growth factor and sildenafil (44) as an inhibitor of cyclic guanosine monophosphate have been used to treat chylous effusion. Etilefrine (45) and Midodrine (46) have been used in cases with refractory chylous effusion through their action on constricting lymphatic smooth muscles. A recent case series has shown improvement in chylous effusion with use of propranolol through proposed action of inhibition of beta-2 adrenergic receptor (47). Chemical pleurodesis, which involves injection of a substance (e.g.: povidine iodine, OK-432) 
in the pleural space, is an additional non-surgical method and has been successfully used in treating refractory congenital chylothorax $(45,48)$.

Persistent chylous effusion may require surgery. Thoracic duct ligation and mechanical pleurodesis have been used for treating resistant postoperative chylothorax in neonates (27). Percutaneous thoracic duct embolization is an alternative to thoracic duct ligation with good response in adults (35) and this procedure has successfully treated two infants with post-operative chylous leaks (49). Pinto et al have described the use of intra nodal injection of ethiodized oil following lymphatic imaging and good response in neonatal chylothorax (42). Pleuro-peritoneal shunt has been suggested in adults who fail thoracic duct ligation or embolization (36) and its use has been reported in seven infants with persistent chylothorax with resolution of symptoms in most; two infants died following sepsis and progressive pulmonary disease (50). Finally, a microsurgical technique called lympho-venous anastomoses which aims to restore lymphatic circulation has been shown to be successful in infants with resistant chylous effusion to reduce the volume $(41,42,51)$.

The strengths and limitations of our study are as follows. To our knowledge, there are limited reports published on developmental outcomes in neonates with chylous effusion. 63\% (12/19) of our cohort had normal developmental scores at one year of age; three had global developmental delay, one each with gross motor delay, dystonic spastic cerebral palsy, autism spectrum disorder and deafness. Resch et al reported normal development in four of the six infants included in their case series assessing congenital chylothorax, with the remaining two children being diagnosed with high functioning autism and delayed development and another having speech delay (31). Limitations of our study include the retrospective design; small sample size, and lack of follow up data beyond 12 months of age. We acknowledge that combining cases of congenital and acquired chylous effusion in our review is a limitation as outcomes and certain aspects of management differ, however provision of comprehensive data for this issue is important

In summary, neonatal chylous effusions are associated with significant morbidity and mortality. Clear evidence-based guidelines need to be considered for the introduction of octreotide in the management of chylous effusions and guarded prognosis to be offered if underlying genetic syndrome/association is present and if there is increased need for mechanical ventilation and oxygenation. We agree with other authors about the need for an international multicenter trial to focus on various aspects of the disease process and to optimize evidence-based management including use of newer modalities in diagnosis and management.

\section{ACKNOWLEDGMENT}

Our sincere thanks to Dr Judy McMichael for providing data on developmental outcomes and Mr Damber Shreshta for neonatal data (NeoBase).

\section{CONFLICT OF INTEREST AND DISCLOSURE}

The authors declare no competing financial interests exist.

\section{REFERENCES}

1. Bellini C, Z Ergaz, M Radicioni, et al: Congenital fetal and neonatal visceral chylous effusions: Neonatal chylothorax and chylous ascites revisited. A multicenter retrospective study. Lymphology 45 (2012), 91-102.

2. Van Straaten, HLM, LJ Gerards, TG Krediet: Chylothorax in the neonatal period. Eur. J. Pediatr. 152 (1993), 2-5.

3. Tutor, JD: Chylothorax in infants and children. Pediatrics 133 (2014), 722-733.

4. Chernick, V, M Reed: Pneumothorax and chylothorax in the neonatal period. J Pediatr. 76 (1970), 624-632.

5. Attar, MA, SM Donn: Congenital chylothorax. Semin. Fetal Neonat. Med. 22 (2017), 234-239.

6. Bialkowski, A, CF Poets, AR Franz: Congenital chylothorax: A prospective nationwide epidemiological study in 
Germany. Arch. Dis. Child Fetal Neonat. Ed. 100 (2015), F169-F172.

7. Efrati, O, A Barak: Pleural effusions in the pediatric population. Pediatr. Rev. 23 (2002), 417-426.

8. Bellini, C, R Cabano, LC De Angelis, et al: Octreotide for congenital and acquired chylothorax in newborns: A systematic review. J. Paediatr. Child Health 54 (2018), 840-847.

9. Geary, B, B Wade, W Wollmann, et al: Laparoscopic repair of chylous ascites. J. Urol. 171 (2004), 1231-1232.

10. Rocha, G, P Fernandes, P Rocha, et al: Pleural effusions in the neonate. Acta Paediatr. 95 (2006), 791-798.

11. Beghetti, M, G La Scala, D Belli, et al: Etiology and management of pediatric chylothorax. J Pediatr. 136 (2000), 653-658.

12. Karagol, BS, A Zenciroglu, S Gokce, et al: Therapeutic management of neonatal chylous ascites: Report of a case and review of the literature. Acta Paediatr. 99 (2010), 13071310.

13. Wu, JM, CT Yao, C Kan, et al: Postoperative chylothorax: Differences between patients who received median sternotomy or lateral thoracotomy for congenital heart disease. $J$. Card. Surg. 21 (2006), 249-253.

14. Gonzalez, R, BS Bryner, DH Teitelbaum, et al: Chylothorax after congenital diaphragmatic hernia. J. Pediatr. Surg. 44 (2009), 1181-1185.

15. Fahimi, H, FP Casselman, MA Mariani, et al: Current management of postoperative chylothorax. Ann. Thorac. Surg. 71 (2001), 448-451.

16. De Angelis, LC, T Bellini, MH Witte, et al: Congenital chylothorax: Current evidencebased prenatal and post-natal diagnosis and management. Lymphology 52 (2019), 108125.

17. Staats, BA, RD Ellefson, LL Budahn, et al: The lipoprotein profile of chylous and non chylous pleural effusions. Mayo Clin. Proc. 55 (1980), 700-704.

18. Buttiker, V, S Fanconi, R Birger: Chylothorax in children: Guidelines for diagnosis and management. Chest 116 (1999), 682-687.

19. Al-Tawil K, Ahmed G, Al-Hathal M, et al: Congenital chylothorax. Am. J. Perinatol. 17 (2000), 121-126.

20. Caserio, S, C Gallego, P Martin, et al: Congenital chylothorax: From foetal life to adolescence. Acta Paediatr. 99 (2010), 1571-1577.
21. Dorsi, M, A Giuseppi, F Lesage, et al: Prenatal factors associated with neonatal survival of infants with congenital chylothorax. J. Perinatol. 38 (2018), 31-34.

22. Mallmann, MR, V Graham, B Rösing, et al: Thoracoamniotic shunting for fetal hydrothorax: Predictors of intrauterine course and postnatal outcome. Fetal Diagn. Ther. 41 (2017), 58-65.

23. Picone, O, A Benachi, L Mandelbrot, et al: Thoracoamniotic shunting for fetal pleural effusions with hydrops. Am. J. Obstet. Gynecol. 191 (2004), 2047-2050.

24. Wei, X, M Meng, G Zou, et al: Zhonghua Fu Chan Ke Za Zhi. 53 (2018), 590-594.

25. White, MK, R Bhat, A Greenough, et al: Neonatal chylothoraces: A 10-year experience in a tertiary neonatal referral centre. Case Rep. Pediatr. (2019) 2019:3903598.

26. Shillitoe, BMJ, J Berrington, N Athiraman: Congenital pleural effusions: 15 years singlecentre experience from North-East England. J. Matern. Fetal Neonatal Med. 31 (2018), 2086-2089.

27. Costa, KM, AK Saxena: Surgical chylothorax in neonates: Management and outcomes. World J. Pediatr. 14 (2018), 110-115.

28. Healy, H, K Gipson, S Hay, et al: Management and outcomes of congenital chylothorax in the neonatal intensive care unit: A case series. Pediatr. Invest. 1 (2017), 21- 25.

29. Fernandez Alvarez, JR, KD Kalache, et al: Management of spontaneous congenital chylothorax: Oral medium-chain triglycerides versus total parenteral nutrition. Am. J. Perinatol. 16 (1999), 415-420.

30. Biewer, ES, C Zürn, R Arnold, et al: Chylothorax after surgery on congenital heart disease in newborns and infants -risk factors and efficacy of MCT-diet. J. Cardiothorac. Surg. 5 (2010), 127.

31. Resch, B, M Halmer, WD Müller, et al: Longterm follow-up of children with congenital chylothorax. Eur. Respir J. 40 (2012), 10601062.

32. Takahashi, Y, Y Kinoshita, T Kobayashi, et al: Management of refractory chylothorax in the neonatal intensive care unit: A 22-year experience. Pediatr Int. (2021) Oct 27. doi: 10.1111/ped.15043. Online ahead of print.

33. Rasiah, SV, J Oei, K Lui: Octreotide in the treatment of congenital chylothorax. J. Paediatr. Child Health 40 (2004), 585-588.

34. Das, A, PS Shah: Octreotide for the treatment 
of chylothorax in neonates. Cochrane Database Syst. Rev. 9 (2010), CD006388.

35. Majdalany, BS, DA Murrey Jr, et al: ACR appropriateness criteria chylothorax treatment planning. Expert Panel on Vascular Imaging and Interventional Radiology. J Am Coll Radiol. 14 (2017), S118-S126. doi:10.1016/j.jacr.2017.02.025

36. Bender, B, V Murthy, RS Chamberlain: The changing management of chylothorax in the modern era. Eur. J. Cardiothorac. Surg. 49 (2016), 18-24.

37. Riley, LE, A Ataya: Clinical approach and review of causes of a chylothorax. Respir. Med. 157 (2019), 7-13.

doi:10.1016/j.rmed.2019.08.014

38. Wiesner, S, E Loch, W Uller, et al: Evaluation of treatment options for postoperative and spontaneous chylothorax in adults. Interact. Cardiovasc. Thorac. Surg. 33 (2021), 527-533. doi:10.1093/icvts/ivab127

39. Itkin, M: Magnetic resonance lymphangiography and lymphatic embolization in the treatment of pulmonary complication of lymphatic malformation. Semin. Intervent. Radiol. 34 (2017), 294-300. doi:10.1055/s-0037-1604301

40. Bellini, C, G Villa, G Sambuceti, et al: Lymphoscintigraphy patterns in newborns and children with congenital lymphatic dysplasia. Lymphology 47 (2014), 28-39.

41. Mihara, M, H Hara, J Shibasaki, et al: Indocyanine green lymphography and lymphaticovenous anastomosis for generalized lymphatic dysplasia with pleural effusion and ascites in neonates. Ann. Vasc. Surg. 29 (2015), 1111-1122. doi:10.1016/j.avsg.2015.02.013

42. Pinto, E, Y Dori, C Smith, et al: Neonatal lymphatic flow disorders: impact of lymphatic imaging and interventions on outcomes. J. Perinatol. 10 (2020), 1038/s41372-020-00771-3.

43. Wiegand, S, G Wichmann, A Dietz: Treatment of lymphatic malformations with the mTOR inhibitor sirolimus: A systematic review. Lymphat. Res. Biol. 16 (2018), 330339. doi:10.1089/lrb.2017.0062.

44. Malleske, DT, BA Yoder: Congenital chylothorax treated with oral sildenafil: A case report and review of the literature. $\mathbf{J}$. Perinatol. 35 (2015), 384-386. doi:10.1038/jp.2015.10.
45. Tomobe, Y, U Mizuguchi, A Shimotakahara, et al: Combination therapy with etilefrine and pleurodesis for refractory congenital chylothorax. Biomed. Hub. 5 (2020), 907-911. doi:10.1159/000509903

46. Tamaoka, S, A Osada, T Kin, et al: Midodrine, an oral alpha-1 adrenoreceptor agonist, successfully treated refractory congenital chylous pleural effusion and ascites in a neonate. Chest 159 (2021), e189e191. doi:10.1016/j.chest.2020.10.071

47. Mitchell, K, A Weiner, P Ramsay, et al: Use of propranolol in the treatment of chylous effusions in infants. Pediatrics 148 (2021), e2020049699. doi:10.1542/peds.2020-049699.

48. Borcyk, K, A Kamil, K Hagerty, et al: Successful management of extremely highoutput refractory congenital chylothorax with chemical pleurodesis using $4 \%$ povidoneiodine and propranolol: A case report. Clin. Case Rep. 6 (2018), 702-708. doi:10.1002/ccr3.1449

49. Itkin, M, G Krishnamurthy, MY Naim, et al: Percutaneous thoracic duct embolization as a treatment for intrathoracic chyle leaks in infants. Pediatrics 128 (2011), e237-e241. doi:10.1542/peds.2010-2016

50. Engum, SA, FJ Rescorla, KW West, et al: The use of pleuroperitoneal shunts in the management of persistent chylothorax in infants. J Pediatr. Surg. 34 (1999), 286-290. doi:10.1016/s0022-3468(99)90192-6.

51. Weissler, JM, EH Cho, PF Koltz, et al: Lymphovenous anastomosis for the treatment of chylothorax in infants: A novel microsurgical approach to a devastating problem. published correction appears in Plast. Reconstr. Surg. 142 (2018), 581.

\author{
Dr. Ajay Prakash Anvekar, FRACP \\ Department of Neonatal Paediatrics \\ Fiona Stanley Hospital \\ Perth, Western Australia, Australia 6009 \\ Tel: 61893401260 \\ Fax: 61893401266 \\ Email: ajayanvekar@yahoo.co.in
}

